\title{
A Distributed LSP Scheme to Reduce Spare Bandwidth Demand in MPLS Networks
}

\author{
Kyu-Seek Sohn, Member, IEEE, Seung Yeob Nam, Member, IEEE, and Dan Keun Sung, Senior Member, IEEE
}

\begin{abstract}
A preplanned path-protection scheme with sufficient spare bandwidth is appropriate for real-time fault restoration in multiprotocol label switching (MPLS) networks. In this case, however, it is important to reduce the amount of spare bandwidth to prevent degradation of network efficiency. A distributed label switched path (D-LSP) scheme is proposed to reduce the amount of spare bandwidth required for protecting against network faults in MPLS networks. The main idea of the proposed D-LSP scheme is to partition traffic into multiple LSPs, each of which is established on a distinct link-disjoint route between each pair of end nodes. The D-LSP scheme is evaluated in terms of the reduction ratio of total network cost in comparison with the conventional LSP scheme. Traffic partitioning in the D-LSP scheme can decrease the statistical multiplexing gain (SMG) obtained by aggregating IP packet flows into an LSP. The tradeoff between spare bandwidth reduction and degradation of SMG due to traffic partitioning is also investigated. The numerical results show that the proposed D-LSP scheme yields the network cost-reduction ratio (NCRR) of at least $29 \%, 27 \%$, and $15 \%$ for the networks where average node degrees are 4.6, 4.4, and 3.2, respectively. The D-LSP scheme shows the similar performance of NCRR in both Markovian traffic and self-similar traffic environments.
\end{abstract}

Index Terms-Distributed label switched path (D-LSP), label switched path (LSP) partitioning, network cost-reduction ratio (NCRR), spare bandwidth reduction, statistical multiplexing gain (SMG).

\section{INTRODUCTION}

$\mathbf{M}$ ULTI-PROTOCOL label switching (MPLS) has been developed for supporting traffic engineering and quality-of-service (QoS) guarantees in Internet backbone networks [1], [2]. MPLS networks provide connection-oriented data transfer services based on label switched paths (LSPs) established between label edge router (LER) pairs. Since a connection-oriented network requires overhead to maintain connections, network response to a change in status or network faults is comparatively slow. This is a major drawback when MPLS is implemented in Internet backbone networks with

Paper approved by T. T. Lee, the Editor for Switching Systems and Network Performance of the IEEE Communications Society. Manuscript received May 7, 2003; revised December 29, 2005. This work was supported by the Korea Research Foundation under Grant KRF-2004-214-D00377, funded by the Government of Korea.

K.-S. Sohn is with the Department of Information and Communications, Hanyang Cyber University, Seoul 133-791, Korea (e-mail: kssohn@ @ycu.ac.kr).

S. Y. Nam is with the Department of Electrical and Computer Engineering, Carnegie Mellon University, Pittsburgh, PA 15213 USA (e-mail: nsyeob@gmail.com).

D. K. Sung is with the Department of Electrical Engineering and Computer Science, Korea Advanced Institute of Science and Technology, Daejeon 305701, Korea (e-mail: dksung@ee.kaist.ac.kr).

Digital Object Identifier 10.1109/TCOMM.2006.877957 very high link speeds using advanced optical transmission and switching technologies.

Preplanned path protection with spare bandwidth is the most appropriate for restoration of MPLS networks in real time due to fast restoration speed [3], [4] and the efficiency of spare capacity [5]. A backup path is pre-established in the preplanned path-protection scheme, with reserved spare bandwidth for each working path.

The cost of establishing a path is the sum of the link costs incurred along the path. The cost of a link is the product of the bandwidth assigned to the link and the link weight representing the state of the link considering congestion, maintenance cost, and usage. Reservation of spare bandwidth on a backup path causes an additional cost and also degrades network efficiency. Minimization of the cost for the backup path can be achieved by either reducing the amount of spare bandwidth or reducing the sum of the link weights along the backup path. In backbone networks transferring a large amount of aggregated traffic with high reliability, links are heavily used and require high maintenance costs. Thus, the link weights are heavy. It is more important to reduce the amount of spare bandwidth for network efficiency than attempting to reduce the link weights in backbone networks.

There have been many studies regarding minimization of backup path costs in which a network-wide cost-minimization problem consists of all working paths and backup paths. The problem is formulated as a multi-commodity flow integer-programming problem, known as NP-hard. Heuristics are required to approximate the optimum solution. Unfortunately, these heuristics are based on a tradeoff between computational complexity and accuracy, and no online heuristics have been found to work effectively in large networks [6], [7].

We propose a distributed LSP (D-LSP) scheme as a new approach to reduce the amount of spare bandwidth and the total network cost in MPLS networks. In the D-LSP scheme, traffic on an LSP between an LER pair is partitioned and distributed over a set of sub-LSPs established on different link-disjoint routes connecting the LER pair. The spare bandwidth required for protecting the traffic of a D-LSP from network failures equals the bandwidth assigned to a sub-LSP, rather than the bandwidth required for the entire LSP traffic. In this paper, "link-disjoint route" is defined as follows. For a given set of routes, they are said to be link-disjoint routes if any two of them share no link with each other. Link-disjoint routes can share some nodes with each other.

Less spare bandwidth is required as more sub-LSPs are used for a given D-LSP. Thus, the backup path cost can be further reduced. However, an increase in the number of sub-LSPs increases the number of links included in the sub-LSPs, and in- 


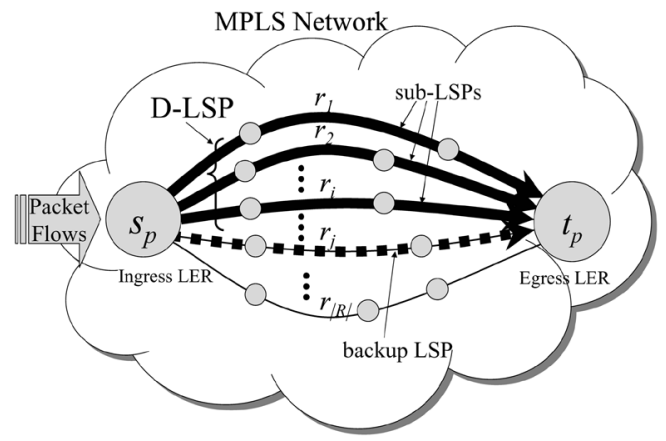

Fig. 1. Model of the proposed D-LSP architecture.

creases the cost of the sub-LSPs. Since the D-LSP cost is defined as the sum of the sub-LSP costs plus the backup LSP cost, there is a tradeoff between the number of sub-LSPs and the amount of spare bandwidth required to minimize the D-LSP cost. We show that the process of searching for the optimum solution is simple, and the network cost is significantly reduced by minimizing the individual D-LSP cost.

Traffic partitioning in the D-LSP scheme may decrease the statistical multiplexing gain (SMG) obtained by aggregating internet protocol (IP) packet flows into an LSP, as compared with the conventional LSP scheme. The tradeoff between spare bandwidth reduction and degradation of SMG in LSP partitioning is also investigated.

The paper is organized as follows. Section II proposes a D-LSP architecture in MPLS networks. Parameters affecting network cost reduction of the D-LSP scheme are defined, and the allocation of sub-LSPs to link-disjoint routes is described in Section III. The SMG is considered in Section IV with optimum LSP partitioning. In Section V, the D-LSP scheme is applied to example network topologies and the cost-reduction effect of the D-LSP scheme is investigated. Conclusions are presented in Section VI.

\section{A D-LSP ARCHITECTURE}

Fig. 1 shows a D-LSP architecture in an MPLS network. A D-LSP consists of a set of sub-LSPs and a backup LSP. SubLSPs of the D-LSP serve the traffic between a pair of LERs. The backup LSP assumes the responsibility to provide resources such as links, labels, and spare bandwidth needed for restoring D-LSP service in the event of failures.

In the D-LSP scheme, each of the sub-LSPs and a backup LSP are established on different link-disjoint routes. Let $R_{p}=\left\{r_{p, 1}, r_{p, 2}, \ldots, r_{p, n}\right\}$ be the set of link-disjoint routes connecting the LER pair of D-LSP $p$, where $n$ is the number of elements of the set $R_{p}$. If we let $\Pi_{p}=\left\{r_{p, i_{1}}, r_{p, i_{2}}, \ldots, r_{p, i_{m}}\right\}$ be the set of link-disjoint routes used for establishing sub-LSPs, where $m=\left|\Pi_{p}\right|, i_{\mu} \in\left\{1,2, \ldots,\left|R_{p}\right|\right\}, 1 \leq \mu \leq m$, then $\Pi_{p} \subset R_{p}$. The notation $|X|$ represents the number of elements of a set $X$. The backup LSP of D-LSP $p$ is established on the link-disjoint route $r_{j} \in R_{p} \backslash \Pi_{p}$.

We assume that a single link failure is the only networkfailure scenario. Since each sub-LSP is established on a different link-disjoint route, at most, a single sub-LSP can be affected by a network failure in each D-LSP. Thus, the amount of spare bandwidth sufficient for a given D-LSP equals to the maximum of the bandwidth demanded by each sub-LSP of the D-LSP.

In MPLS networks, an IP packet flow can be defined as the set of packets which have the same destination IP address. An ingress LER aggregates a set of incoming packet flows forwarded to the same egress LER into an LSP in the conventional LSP scheme. The ingress LER of a D-LSP groups the incoming IP packet flows into several groups, each of which is assigned to a distinct sub-LSP. It is assumed that splitting a packet flow into different groups is not allowed. Thus, the D-LSP scheme does not suffer from the problem of packet reordering.

\section{OPTIMUM LSP PARTITIONING}

LSP partitioning is used to reduce the amount of spare bandwidth required in the D-LSP scheme.

LSP partitioning is a process for selecting an optimum set of link-disjoint routes to which sub-LSPs and the backup LSP are assigned. Distribution of the required and the spare bandwidth over the selected link-disjoint routes for minimization of the D-LSP cost is also achieved by LSP partitioning.

The cost of a path is defined as the sum of the products of the bandwidth assigned to a path and the weight of each link along the path. We assume that every link has a weight of unity. Thus, the cost of a path can be represented by the product of the bandwidth and the number of links included in the path.

We can now develop the theoretical basis and algorithms for LSP partitioning. The subscript $p$ used previously is dropped from notations, since we consider a single D-LSP hereafter.

\section{A. Definitions}

Let $D$ be the bandwidth required for carrying the incoming packet flows of a D-LSP, and let $w_{r}$ be the bandwidth that should be assigned to a sub-LSP established on a link-disjoint route $r \in \Pi$ for carrying the group of packet flows assigned to the sub-LSP

$$
D=\sum_{r \in \Pi} w_{r}
$$

The cost of a sub-LSP established on a link-disjoint route $r$ is the product of $w_{r}$ and the sum of weights of links comprising $r$. Since the weight of every link is unity (assumed in Section II), the sum of the weights of links included in a link-disjoint route is equal to the length of the route. The length of the route is the number of links included in the route. The D-LSP cost required for establishing a reliable D-LSP is the sum of the working cost and the backup LSP cost. The working cost of a D-LSP required for carrying the traffic is expressed as

$$
C_{\mathrm{work}}=\sum_{r \in \Pi} \sum_{l \in r} c_{\mathrm{work}}(r, l)=\sum_{r \in \Pi} w_{r}|r|
$$

where $c_{\mathrm{work}}(r, l)$ is the cost of the link $l$ originating from the sub-LSP established on the route $r$, and $|r|$ represents the length of the route $r$. 
In order to guarantee restoration after a single link failure, the amount of spare bandwidth reserved on the backup LSP is determined as

$$
s=\max _{r \in \Pi}\left(w_{r}\right) .
$$

The cost of the backup LSP established on $r_{j}$ with the spare bandwidth is defined as

$$
C_{\text {spare }}=\sum_{l \in r_{j}} c_{\text {spare }}\left(r_{j}, l\right)=s\left|r_{j}\right| \quad \text { for } r_{j} \in R \backslash \Pi
$$

where $c_{\text {spare }}(r, l)$ is the portion of the cost of the link $l$ used for backing up the D-LSP.

\section{B. Problem Statement}

We investigate the problem of minimization of the D-LSP cost, $C_{\mathrm{dlsp}}$ used by the D-LSP in order to provide reliable services. The objective function is expressed as

$$
\min . C_{\mathrm{dlsp}}=C_{\text {working }}+C_{\text {spare }} \text {. }
$$

Equations (1)-(4) are the constraints of the objective function (5). From (3), the amount of spare bandwidth is minimized when the bandwidth is uniformly distributed over a given number of sub-LSPs. The amount of spare bandwidth can be further reduced as the number of sub-LSPs increases. However, from (2), the working cost of the D-LSP can be reduced by allocating more bandwidth to the shorter sub-LSPs, and thus by establishing a single sub-LSP on the shortest link-disjoint route between the LER pair. The parameters affecting the minimization of the D-LSP cost $\left(C_{\mathrm{dlsp}}\right)$ are the number of sub-LSPs $(|\Pi|)$, the set of link-disjoint routes on which the sub-LSPs are established $(\Pi)$, and distribution of the bandwidth demand among the sub-LSPs and the backup LSP.

From (3) and (4), the cost of the LSP backup depends on the bandwidth distributed over the sub-LSPs and how the set of link-disjoint routes is selected. The solution of the objective function (5) is computationally complex if the dependency is not dissolved. In order to simplify the problem, we first consider the set of link-disjoint routes used for establishing the sub-LSPs, the bandwidth distribution over the sub-LSPs, and the number of the required sub-LSPs, in sequence.

\section{Bandwidth Distribution}

Let $\mathbf{r}$ be a sorted vector of link-disjoint routes connecting the LER pair of a D-LSP. Vector $r$ is defined as

$$
\begin{aligned}
\mathbf{r}=\left(r_{1}, r_{2}, \ldots, r_{|R|}\right), & \left|r_{i}\right|>0 \quad \text { for } i=1, \ldots,|R|, \\
& \left|r_{i}\right| \leq\left|r_{j}\right| \quad \text { if } i<j .
\end{aligned}
$$

Elements of $\mathbf{r}$ are sorted in ascending order based on length.

We define the bandwidth distribution vector $\mathbf{w}$ that represents the distribution of bandwidth over the sub-LSPs in a D-LSP as

$$
\mathbf{w}=\left(w_{1}, w_{2}, \ldots, w_{|R|}\right), w_{i} \geq 0 \quad \text { for } i: r_{i} \in \mathbf{r}, \sum_{i=1}^{|R|} w_{i}=D
$$

where $w_{i}$ is the bandwidth assigned to the sub-LSP established on the route $r_{i} \in \mathbf{r}$.

For a given LER pair, we define the set of all possible bandwidth distribution vectors $A$ as

$$
A=\{\mathbf{w}\} .
$$

From (7), $A$ represents a hyperplane.

Lemma 1: For nonnegative real numbers $a_{1}, a_{2}\left(a_{1} \geq a_{2}\right)$ and $b_{1}, b_{2}\left(b_{1} \geq b_{2}\right)$, the following relation is valid:

$$
a_{1} b_{1}+a_{2} b_{2} \geq a_{1} b_{2}+a_{2} b_{1} .
$$

0.

Proof: $a_{1} b_{1}+a_{2} b_{2}-a_{1} b_{2}-a_{2} b_{1}=\left(a_{1}-a_{2}\right)\left(b_{1}-b_{2}\right) \geq$

Lemma 2: If $\mathbf{w}^{\prime}=\left(w_{1}^{\prime}, w_{2}^{\prime}, \ldots, w_{|R|}^{\prime}\right)$ is derived from $\mathbf{w} \in$ $A$ by sorting the elements of $\mathbf{w}$ in descending order, such that $w_{1}^{\prime} \geq w_{2}^{\prime} \geq \cdots \geq w_{|R|}^{\prime}$, then $C_{\mathrm{dlsp}}\left(\mathbf{w}^{\prime}\right) \leq C_{\mathrm{dlsp}}(\mathbf{w})$.

Proof: This lemma can be proved easily using Lemma 1, and we omit the detailed derivation.

We define $B \subset A$ as

$$
\begin{aligned}
& B=\left\{\left(w_{1}, w_{2}, \ldots, w_{|R|}\right):\right. \\
& \frac{D}{|R|} \leq w^{*} \leq D, K=\left\lfloor\frac{D}{w^{*}}\right\rfloor, w_{i}=w^{*} \quad \text { for } i=1, \ldots, K \\
& \left.w_{K+1}=D-K w^{*}, w_{j}=0 \text { for } j>K+1\right\} .
\end{aligned}
$$

Bandwidth distribution vectors included in $B$ represent that the bandwidth is distributed uniformly with a constant value of $w^{*}$ among all the sub-LSPs with the possible exception of the $K+$ 1th sub-LSP. Set $B$ represents a curve on the hyperplane represented by the set $A$.

Theorem 1: For all $\mathbf{w} \in A$, there exists $\mathbf{w}^{\prime} \in B$ such that $C_{\mathrm{dlsp}}(\mathbf{w}) \geq C_{\mathrm{dlsp}}\left(\mathbf{w}^{\prime}\right)$, where $A$ and $B$ are defined as (8) and (9), respectively.

The proof of Theorem 1 is shown in Appendix A.

Thus, by Theorem 1 , for given $R$ and $\Pi$, the vector $\mathbf{w}^{\prime}$ minimizing $C_{\mathrm{dlsp}}$ always exists in $B$ and has the following form:

$$
\begin{aligned}
& \mathbf{w}^{\prime}=(\overbrace{w^{*}, w^{*}, \ldots, w^{*}}^{K}, \alpha, 0, \ldots, 0), \\
& \text { such that } 0 \leq \alpha<w^{*}, \quad K w^{*}+\alpha=D \text {. }
\end{aligned}
$$

Thus, the space to be searched for the optimum parameters has been reduced from $A$ (hyperplane) to $B$ (curved line on the hyperplane). Now, we show that we can find the optimum working parameters by evaluating a sequence of a finite number of vectors in $B$ (a finite number of points on the line) rather than the entire infinite set $B$.

Theorem 2: For a given D-LSP, at least one of the following relations is true:

$$
\begin{aligned}
& C_{\mathrm{dlsp}}\left(\left(w_{1}, w_{2}, \ldots, w_{K}, \alpha, 0, \ldots, 0\right)\right) \\
& \quad \geq C_{\mathrm{dlsp}}\left(\left(w_{1}+\frac{\alpha}{K}, w_{2}+\frac{\alpha}{K}, \ldots, w_{K}+\frac{\alpha}{K}, 0, \ldots, 0\right)\right)
\end{aligned}
$$

$C_{\mathrm{dlsp}}\left(\left(w_{1}, w_{2}, \ldots, w_{K}, \alpha, 0, \ldots, 0\right)\right)$

$$
>C_{\mathrm{dlsp}}\left(\left(w_{1}^{\prime}, w_{2}^{\prime}, \ldots, w_{K+1}^{\prime}, 0, \ldots, 0\right)\right)
$$


where $0 \leq \alpha<(D) /(K+1), w_{1}=w_{2}=\ldots=w_{K}=$ $w^{*}, w_{1}^{\prime}=w_{2}^{\prime}=\cdots w_{K+1}^{\prime}=(D) /(K+1)$.

Proof of Theorem 2 is shown in Appendix B.

Finally, for a given D-LSP, $C_{\mathrm{dlsp}}(\mathbf{w})$ can be minimized with $\mathrm{w}$ having the following form:

$$
\begin{aligned}
\mathbf{w}= & \left(w_{1}, w_{2}, \ldots, w_{K}, 0, \ldots, 0\right), \\
& \text { where } w_{i}=w_{j} \forall i, j(\leq K) \text { and } \sum_{i=1}^{K} w_{i}=D .
\end{aligned}
$$

By Theorem 2, we can minimize the D-LSP cost by distributing the bandwidth demand uniformly among sub-LSPs regardless of the position of the backup LSP among the $(K+1)$ shortest linkdisjoint routes. The bandwidth distribution vector is specified only by the number of sub-LSPs. Thus, the remaining problem is determination of the optimum number of sub-LSPs in the given D-LSP.

\section{Optimum Number of Sub-LSPs}

The D-LSP cost is minimized using the optimum number of sub-LSPs. Let $r_{\text {backup }}$ be the link-disjoint route on which the backup LSP is established. From (2)-(5), and (13), the D-LSP cost is expressed as a function of the number of sub-LSPs, $k$

$$
C_{\text {dlsp }}(k)=\frac{D}{k}\left\{\sum_{i=1}^{k}\left|r_{i}\right|+\left|r_{\text {backup }}\right|\right\}
$$

where $r_{i} \in R(i=1, \ldots, k)$ is the link-disjoint route on which the sub-LSP is established. In (14), $D$ is given and $\left|r_{i}\right|$ is determined by a given network topology.

Theorem 3: If there is a $\hat{k}$ such that $C_{\mathrm{dlsp}}(\hat{k}) \leq C_{\mathrm{dlsp}}(\hat{k}+1)$, then $C_{\mathrm{dlsp}}(\hat{k}) \leq C_{\mathrm{dlsp}}(j)$ for all $j \geq \hat{k}$.

Proof of Theorem 3 is shown in Appendix C.

\section{E. A D-LSP Cost Minimization Algorithm}

By Theorems 2 and 3, there exist $\Pi$ and $\mathbf{w}$ such that the D-LSP cost for a given D-LSP is minimized in the network. The algorithm for establishing the D-LSP with the minimum cost is described in Table I.

In Phase 1, the algorithm to determine the minimum cost of D-LSP creates the vector of link-disjoint routes, $\mathbf{r}$ defined as (6). The algorithm selects routes from $\mathbf{r}$ and calculates the D-LSP cost after assigning sub-LSPs and the backup LSP to the selected routes in Phase 2. If the current D-LSP cost is smaller than the previous D-LSP cost, the algorithm increases the number of selected routes by one and repeats Phase 2. Otherwise, the algorithm decides that the D-LSP cost reaches a minimum and determines the optimum parameters used for establishing the D-LSP with the minimum cost in Phase 3.

\section{SMG AND LSP PARTITIONING}

Thus far, we did not consider statistical multiplexing of traffic flows in the LSP partitioning problem. In this section, we investigate an LSP partitioning problem considering statistical multiplexing of traffic flows.
TABLE I

ALGORITHM FOR ESTABLISHING A D-LSP WiTh THE Minimum COST

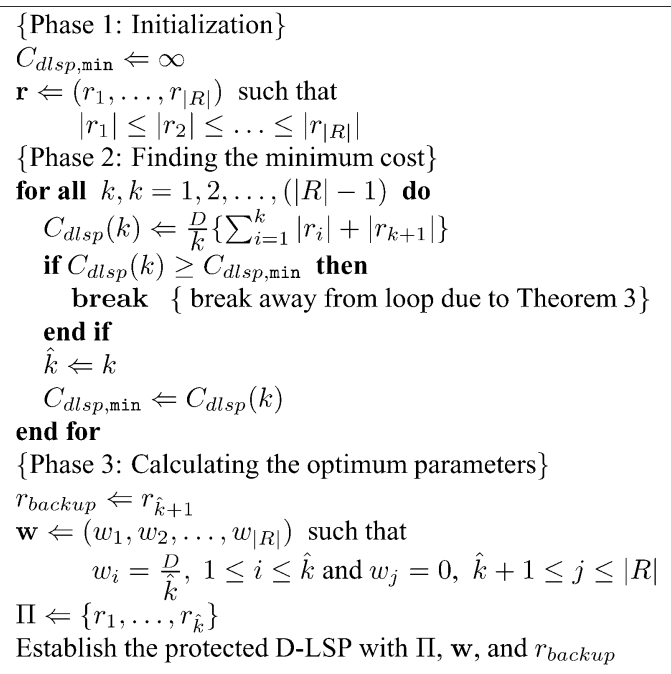

\section{A. Statistical Multiplexing Gain}

SMG can be different depending on the characteristics of traffic flows. We consider two types of models for traffic flows. The first one is the two-state fluid-flow model [10], [11] for Markovian traffic and the second is the fractional Brownian motion model for self-similar traffic.

For Markovian traffic, the SMG is defined as the ratio of the sum of the peak rates of flows to the effective bandwidth that is sufficient for the sub-LSP to guarantee QoS requirements [9]. The SMG obtained in the $i$ th sub-LSP is represented as follows:

$$
\gamma_{i}^{\mathrm{smg}}\left(n_{i}\right)=\frac{\sum_{j=1}^{n_{i}} r_{j}}{w_{i}^{\text {eff }}\left(n_{i}\right)}
$$

where $n_{i}$ is the number of IP packet flows aggregated into the $i$ th sub-LSP, $w_{i}^{\text {eff }}$ is the effective bandwidth demanded by the $i$ th sub-LSP, and $r_{j}$ is the peak rate of the $j$ th packet flow in the sub-LSP. As shown in (15), the SMG in a sub-LSP is a function of the number of packet flows aggregated in the sub-LSP. For Markovian traffic, packet flows belonging to a sub-LSP are modeled as an ON-OFF traffic characterized by 3 -tuple $(r, b, \rho)$, where $r$ is the peak rate, $b$ is the mean burst length, and $\rho$ is the packet-flow source use. The durations of ON and OFF states are exponentially distributed. It is assumed that all packet-flow sources are independent of each other and have identical characteristics, that is, the same $r, b$, and $\rho$. We assume that the same packet-loss rate and delay are required at each link, then, the effective bandwidth $w_{i}^{\text {eff }}$ of a sub-LSP in which $n_{i}$ flows are aggregated is given by [11]

$$
w_{i}^{\mathrm{eff}}\left(n_{i}\right)=\frac{\left(\alpha b(1-\rho)+\rho n_{i} d\right) r n_{i}}{\alpha b(1-\rho)+d n_{i}}
$$

where $\alpha=\log (1 / \epsilon), d$ is the desired queueing delay, and $\epsilon$ is the desired packet-loss ratio. In this equation, we assume that the QoS requirements of packet-loss rate and packet delay are identical at each LSR node. 
However, the peak rate is usually not defined in advance for real internet traffic traces because the use of traffic policing or shaping is not popular yet. Thus, it may not be easy to characterize internet traffic with 3 tuple of parameters $(r, b, \rho)$. Instead, today's IP traffic is known to exhibit two important properties: self-similarity and long-range dependence [12]-[14]. Thus, we consider self-similar traffic as the second model of internet traffic. Since fractional Brownian motion is widely used for representing the traffic model with these properties, we consider input traffic patterns of fractional Brownian motion type.

For self-similar traffic, we consider only the delay performance for the QoS requirements since many applications and services provided in the internet these days are very sensitive to delay. For a single-server queueing system, if we let $C, Q$, and $d$ denote the service rate for the interested set of flows, the queue length in steady state, and the mean delay constraint, then the required QoS is expressed as

$$
E[Q] \leq C d
$$

The effective bandwidth $w^{e}$ is defined as the minimum value of the service rate $C$ which satisfies the above relation.

We need to note that in this case, the SMG can not be expressed as (15) because the peak rate is not defined in the fractional Brownian motion traffic flow. Instead, we define the SMG in the following way (as in [15]):

$$
I(n)=\frac{n \times w^{e}(1)}{w^{e}(n)}
$$

where $n$ is the number of self-similar traffic flows aggregated into the sub-LSP, and $w^{e}(i)$ is the effective bandwidth when $i$ flows are aggregated into the sub-LSP. We assume that all traffic flows are independent of each other and have identical characteristics. We assume that the same average delay is required at each link, then the effective bandwidth $w^{e}(n)$ of a sub-LSP is given as the solution of the following equation [15]:

$$
w^{e}(n)=n m+n^{1 / 2 H} \sigma^{1 / H} \frac{\left(E\left[Q^{*}\right] / d\right)^{p}}{w^{e}(n)^{p}}
$$

where $m$ is the mean rate of each flow, $H$ is the Hurst parameter of each flow, $\sigma^{2}$ is the variance of the traffic rate, $p=1 / H-1$, and $Q^{*}$ is the queue length for a reference queueing system, where $\sigma=1$ and $C-m=1$. The value of $w^{e}(n)$ can be obtained in an iterative way from the above relation [15]. The Hurst parameter $H$ can take a value from $(0,1)$, and when $H$ is in $(1 / 2,1)$, the traffic is long-range dependent.

Figs. 2 and 3 show typical curves of SMG obtained from (15) and (17) as a function of the number of packet flows, respectively. These SMG curves increase monotonically as the number of packet flows increases. The monotonicity of the typical SMG curves provides the inspiration of the possiblity that the LSP partitioning problem can be solved analytically.

In this section, we investigate partitioning of packet flows considering SMG. Although we consider partitioning on the

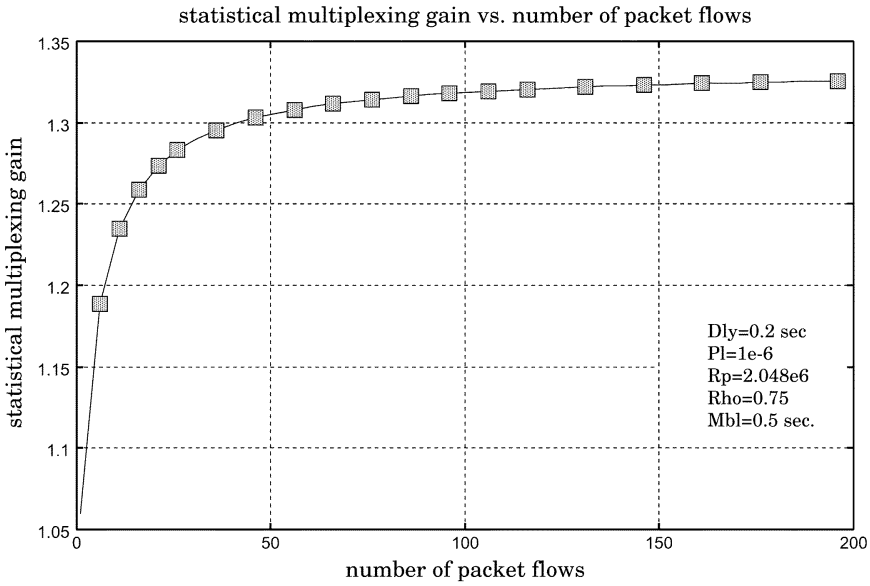

Fig. 2. Typical curve of SMG of ON/OFF traffic versus the number of packet flows $\left(d=0.2 \mathrm{~s}, \epsilon=10^{-6}, r=2.048 \mathrm{Mb} / \mathrm{s}, \rho=0.75\right.$, and $\left.b=0.5 \mathrm{~s}\right)$.

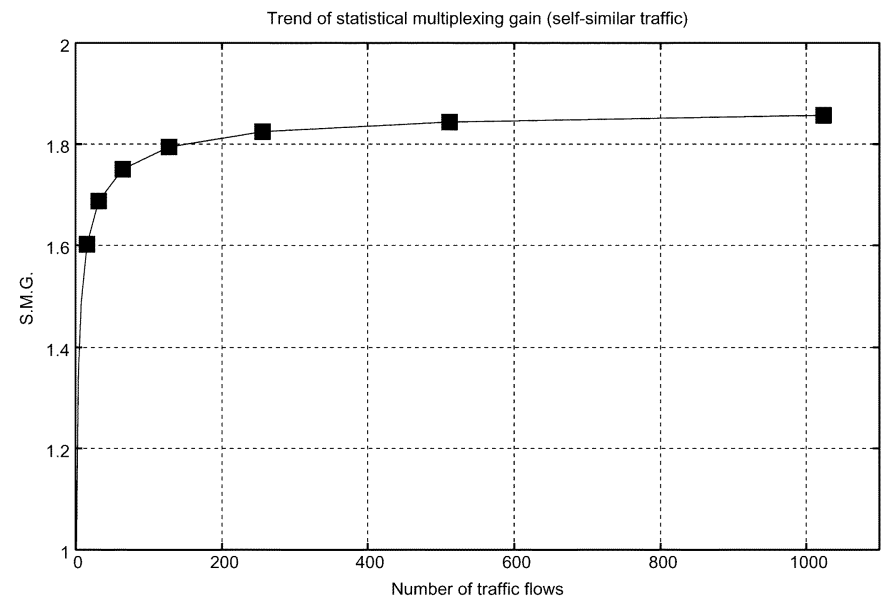

Fig. 3. Typical curve of SMG of self-similar traffic versus the number of packet flows $\left(H=0.8, m=1 \mathrm{Mb} / \mathrm{s}, \sigma=0.4 \mathrm{~m}, Q^{*}=1.12\right.$, and $\left.d=0.01\right)$.

packet-flow level, we do not allow partitioning of a flow into packet-level subgroups in order to prevent the packet-reordering problem. Thus, each sub-LSP can not have continuous bandwidth as in the previous section, but it can have only a discrete number of bandwidth values, depending on the number of the corresponding packet flows. Thus, we use the total number of packet flows as the constraint instead of (1) in this case.

\section{B. Optimum LSP Partitioning for Markovian Traffic}

We first consider an optimum LSP partitioning problem for Markovian traffic. We find the optimum partitioning of total $N$ flows into multiple groups $\mathbf{n}=\left(n_{1}, n_{2}, \ldots, n_{|R|}\right)$, each of which is allocated to a distinct sub-LSP, and $\sum_{i=1}^{|R|} n_{i}=N$ that minimizes the cost function given by

$$
C_{\mathrm{dlsp}}(\mathbf{n})=\sum_{i=1}^{|n|} w^{\mathrm{eff}}\left(n_{i}\right)\left|r_{i}\right|+\left(\max _{1 \leq i \leq|R|} w^{\mathrm{eff}}\left(n_{i}\right)\right)\left|r_{z}\right|
$$

where $\left\{\left|r_{i}\right|\right\}$ is sorted in an ascending order such that $0<\left|r_{1}\right| \leq$ $\left|r_{2}\right| \leq \cdots, r_{z}$ is the link-disjoint route on which the backup LSP is established, and $w^{\text {eff }}\left(n_{i}\right)$ is defined as (16). 
If we let $\tilde{A}$ denote the set of all possible partitioning of total $N$ flows, then $\tilde{A}$ is expressed as

$$
\begin{aligned}
\tilde{A} & =\left\{\left(n_{1}, n_{2}, \ldots, n_{|R|}\right): \sum_{i=1}^{|R|} n_{i}\right. \\
& \left.=N, n_{j} \text { is a nonnegative integer, for } j=1, \ldots,|R|\right\} .
\end{aligned}
$$

Lemma 3: For $\mathbf{n} \in \tilde{A}$, if we obtain $\mathbf{n}^{\prime}=\left(n_{1}^{\prime}, n_{2}^{\prime}, \ldots, n_{|R|}^{\prime}\right)$ by sorting the elements of $\mathbf{n}$ in descending order such that $n_{1}^{\prime} \geq$ $n_{2}^{\prime} \geq \cdots \geq n_{|R|}^{\prime}$, then $C_{\mathrm{dlsp}}\left(\mathbf{n}^{\prime}\right) \leq C_{\mathrm{dlsp}}(\mathbf{n})$.

Proof: This lemma can be proved easily using Lemma 1 .

In order to reduce the possible range of the optimum solutions, we introduce $\tilde{B}$, a subset of $\tilde{A}$, which is defined as

$$
\begin{aligned}
\tilde{B}= & \left\{\left(n_{1}, n_{2}, \ldots, n_{|R|}\right): n^{*}\right. \text { is an integer in } \\
& {\left[\frac{N}{|R|}, N\right], \quad K=\left\lfloor\frac{N}{n^{*}}\right\rfloor } \\
& n_{i}=n^{*} \text { for } i=1, \ldots, K, n_{K+1}=N-K n^{*} \\
& \left.n_{j}=0 \text { for } j>K+1\right\} .
\end{aligned}
$$

Now we show that the optimum partitioning can be found in the set $\tilde{B}$. The effective bandwidth defined in (16) has the following property.

Lemma 4: For positive integers $a_{1}$ and $a_{2}\left(a_{1} \geq a_{2}\right)$

$$
w^{\mathrm{eff}}\left(a_{1}\right)+w^{\mathrm{eff}}\left(a_{2}\right)>w^{\mathrm{eff}}\left(a_{1}+1\right)+w^{\mathrm{eff}}\left(a_{2}-1\right) .
$$

Proof: From (16), we can obtain the relation shown in the equation at the bottom of the page. Since $\rho<1$ and $a_{1}-a_{2}+1>$ $0, w^{\mathrm{eff}}\left(a_{1}\right)+w^{\mathrm{eff}}\left(a_{2}\right)-w^{\mathrm{eff}}\left(a_{1}+1\right)-w^{\mathrm{eff}}\left(a_{2}-1\right)>0$.

Using Lemmas 3 and 4 , we can prove the following theorem.

Theorem 4: For each $\mathbf{n} \in \tilde{A}$, there exists a vector $\mathbf{n}^{\prime} \in \tilde{B}$ such that $C_{\text {dlsp }}\left(\mathbf{n}^{\prime}\right) \leq C_{\mathrm{dlsp}}(\mathbf{n})$.

Proof: The proof is given in Appendix D.

By Theorem 4, we can find the optimum partitioning of $N$ in set $\tilde{B}$ instead of the larger set $\tilde{A}$. We define a function $C_{\text {dlsp }}^{\prime}(n)$ for an element $\mathbf{n}^{\prime}=(n, \ldots, n, \alpha, 0, \ldots, 0)(0 \leq \alpha<n)$ in $\tilde{B}$ as follows:

$$
C_{\mathrm{dlsp}}^{\prime}(n)=C_{\mathrm{dlsp}}((n, \ldots, n, \alpha, 0, \ldots, 0)) .
$$

The optimum partitioning can be obtained by evaluating $C_{\text {dlsp }}^{\prime}(n)$ for an integer $n \in[N /|R|, N]$. Thus, the calculation complexity is $O(N)$.

We introduce two sets $\mathcal{N}_{0}$ and $\mathcal{N}_{1}$ that are determined as

$$
\begin{aligned}
& \mathcal{N}_{0}=\left\{\left\lfloor\frac{N}{k}\right\rfloor: k=1,2, \ldots,|R|\right\} \\
& \mathcal{N}_{1}=\left\{\left\lfloor\frac{N}{k+1}\right\rfloor+1: k=1,2, \ldots,|R|-1\right\} .
\end{aligned}
$$

We can reduce the complexity of the algorithm to find an optimum partitioning by the following theorem.

Theorem 5: $\mathcal{N}_{0} \cup \mathcal{N}_{1}$ has an integer $n=n^{*}$ which minimizes $C_{\text {dlsp }}^{\prime}(n)$.

Proof: The proof is given in Appendix E.

Thus, Theorem 5 implies that the optimum partitioning can be found by checking the value of the cost function $C_{\mathrm{dlsp}}^{\prime}(n)$ at $2|R|-1$ points in $\mathcal{N}_{0} \cup \mathcal{N}_{1}$. Since $|R|$ is usually much smaller than $N$, Theorem 5 reduces the complexity of the solution algorithm from $O(N)$ to $O(|R|)$. The complexity no longer depends on $N$.

\section{Optimum LSP Partitioning for Self-Similar Traffic}

In this subsection, we are considering the same partitioning problem as the previous subsection, but we consider a different traffic type, i.e., self-similar traffic. The cost function is also defined as (19), and Lemma 3 is still valid in this case. But the effective bandwidth is determined in a different way from (18). Since Lemma 4 is dependent on the detailed structure of the effective bandwidth equation, we need to prove this relation for the new effective bandwidth equation. Before proving it, we suggest a condition which guarantees the relation of Lemma 4.

Lemma 5: Let $f(x)$ be twice differentiable on $(a, b)$, and $f^{\prime}(x)$ is continuous on $[a, b]$. If $f^{\prime \prime}(x)<0$ for $x$ in $(a, b)$, then for positive numbers $a_{1}$ and $a_{2}\left(a+1<a_{2} \leq a_{1}<b-1\right)$, we have

$$
f\left(a_{1}\right)+f\left(a_{2}\right)>f\left(a_{1}+1\right)+f\left(a_{2}-1\right) .
$$

Proof: By the mean-value theorem, there must be some number $a_{1}^{\prime}$ in $\left(a_{1}, a_{1}+1\right)$ such that

$$
f\left(a_{1}+1\right)-f\left(a_{1}\right)=f^{\prime}\left(a_{1}^{\prime}\right) .
$$

By the same reason, there is some number $a_{2}^{\prime}$ in $\left(a_{2}-1, a_{2}\right)$ such that

$$
f\left(a_{2}\right)-f\left(a_{2}-1\right)=f^{\prime}\left(a_{2}^{\prime}\right) .
$$

By the condition that $f^{\prime \prime}(x)<0, f^{\prime}(x)$ is decreasing in $(a, b)$. Thus, we have

$$
f^{\prime}\left(a_{1}^{\prime}\right)<f^{\prime}\left(a_{2}^{\prime}\right)
$$

since $a_{2}^{\prime}<a_{2} \leq a_{1}<a_{1}^{\prime}$. Substituting (20) and (21) into (22) completes the proof.

Thus, we only need to show that the effective bandwidth defined in (18) satisfies the condition of Lemma 5 in order to show that it also satisfies the relation in Lemma 4.

$$
\begin{aligned}
w^{\mathrm{eff}}\left(a_{1}\right)+w^{\mathrm{eff}}\left(a_{2}\right)-w^{\mathrm{eff}}\left(a_{1}+1\right) & -w^{\mathrm{eff}}\left(a_{2}-1\right) \\
& =\frac{\alpha^{2} b^{2}(1-\rho)^{3} r d\left(a_{1}-a_{2}+1\right)\left(2 \alpha b(1-\rho)+d\left(a_{1}+a_{2}\right)\right)}{\left(\alpha b(1-\rho)+d a_{1}\right)\left(\alpha b(1-\rho)+d a_{2}\right)\left(\alpha b(1-\rho)+d\left(a_{1}+1\right)\right)\left(\alpha b(1-\rho)+d\left(a_{2}-1\right)\right)}
\end{aligned}
$$




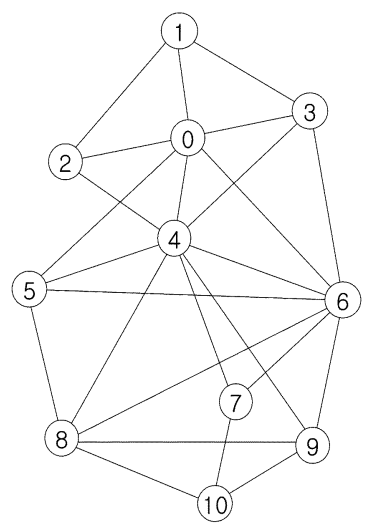

(a)

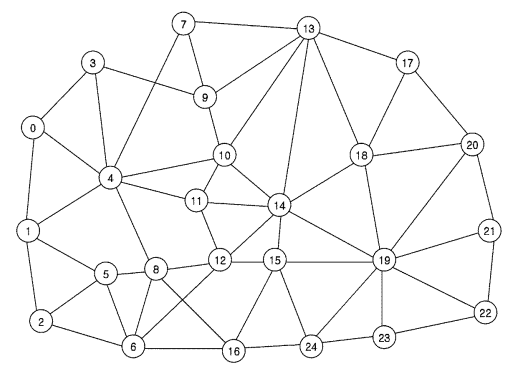

(b)

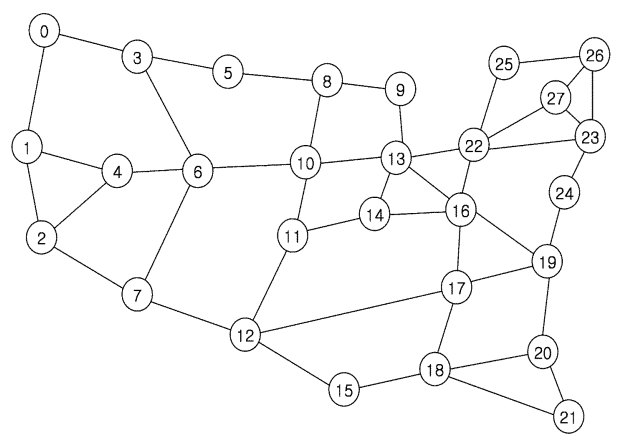

(c)

Fig. 4. Example network topologies. (a) NJ-LATA network. (b) Toronto MAN. (c) US-Longhaul network.

Lemma 6: For positive integers $a_{1}$ and $a_{2}\left(a_{1} \geq a_{2}\right)$

$$
w^{e}\left(a_{1}\right)+w^{e}\left(a_{2}\right)>w^{e}\left(a_{1}+1\right)+w^{e}\left(a_{2}-1\right) .
$$

Proof: The proof is given in Appendix F.

Now we can derive the same result for the self-similar traffic as that for Markovian traffic obtained in the previous subsection.

Theorem 6: $\mathcal{N}_{0} \cup \mathcal{N}_{1}$ has an integer $n=n^{*}$ which minimizes $C_{\mathrm{dlsp}}^{\prime}(n)$

Proof: The proof is given in Appendix G.

\section{NUMERICAL EVALUATION}

\section{A. Example Networks}

In order to evaluate the spare bandwidth reduction effect of the D-LSP scheme in various network and traffic environments, we apply the proposed D-LSP scheme to three example network topologies for Markovian and self-similar traffic models. Network topologies are shown in Fig. 4. These example network topologies are modeled on the LATA telephone service network of New Jersey, USA (NJ-LATA) [16], the metropolitan area network (MAN) of Toronto, Canada [17], and the long-haul network of the USA (US-Longhaul).

Network size is generally characterized by the number of nodes in the network. The complexity of a network is defined as the average degree of nodes in the network. In the NJ-LATA, the Toronto-MAN, and the US-Longhaul, the number of nodes is 10,25 , and 28 , respectively, with a corresponding average node degree of 4.6, 4.4, and 3.2.

We use the algorithms described in [18] for setting up the linkdisjoint routes between an LER pair. LER pairs are randomly chosen from the set of possible node pairs in the network.
For the Markovian traffic model, every traffic consists of 5000 identical and independent packet flows. Each packet flow is modeled with an ON-OFF traffic with a peak rate of $2.048 \mathrm{Mb} / \mathrm{s}$, a burst length of $0.5 \mathrm{~s}$, a packet-loss requirement of $10^{-6}$, a source use of 0.75 , and a delay requirement of $0.2 \mathrm{~s}$.

And for the self-similar traffic model, every traffic consists of 10000 identical and independent packet flows. Each packet flow is modeled with a fractional Braunian motion flow with Hurst parameter of 0.8 , mean bit rate of $1 \mathrm{Mb} / \mathrm{s}$, queue length of 1.12 , and the delay of $0.01 \mathrm{~s}$. The standard deviation of the traffic rate is $0.4 \mathrm{Mb} / \mathrm{s}$.

For numerical evaluation, the effect of statistical multiplexing is considered on each link. The effective bandwidth of each sub-LSP is calculated by using (16) and (18) for Markovian and self-similar traffic, respectively. SMG is calculated by using (15) and (17) for Markovian and self-similar traffic, respectively. The traffic partitioning function is based on Algorithm I.

\section{B. Performance Indicator}

In order to quantitatively evaluate the bandwidth reduction effect of the D-LSP scheme, we define a performance indicator (PI) as follows:

$$
\gamma=1-\frac{\sum_{p \in P} C_{\mathrm{dlsp}, p}}{\sum_{p \in P} C_{\mathrm{lsp}, p}}
$$

where $P$ is the set of LER pairs in the network, $C_{\mathrm{dlsp}, p}$ and $C_{\mathrm{lsp}, p}$ are the costs of D-LSP and the conventional LSP established between an LER pair $p$, respectively. PI is the network cost-reduction ratio (NCRR) between the conventional LSP scheme and the D-LSP scheme.

\section{Optimum Number of Sub-LSPs}

Figs. 5 and 6 show the typical D-LSP cost curves for various number of sub-LSPs carrying Markovian traffic or self-similar traffic. The number of sub-LSPs means the partitioning degree of D-LSP established between each node pair.

\section{Network Cost-Reduction Ratio}

In Fig. 5(a), curves (A) and (B) show the D-LSP cost trends of D-LSPs established between node pairs $(14,4)$ and $(19,4)$, respectively, in Toronto-MAN, where Markovian traffic transferred. These two node pairs have the same number (7) of linkdisjoint routes, but different average route lengths (3.9 and 5.3 links, respectively). The level of D-LSP cost is proportional to the average length of the link-disjoint routes between the node pair. Curve (C) of Fig. 5(a) shows the case of node pair $(6,13)$ having a smaller number (5) of link-disjoint routes.

Curves (A), (B), and (C) of Fig. 5(b) shows the D-LSP cost curves of the NJ-LATA, the Toronto-MAN, and the US-Longhaul, respectively, where all the networks serve Markovian traffic.

Due to Theorem 3, in Figs. 5(a) and (b), each curve has a single minimum point at which the D-LSP cost is minimized. The optimum number of sub-LSPs (i.e., $|\Pi|$ or the optimum partitioning degree) corresponds to the minimum D-LSP cost in the network where Markovian traffic is offered. 


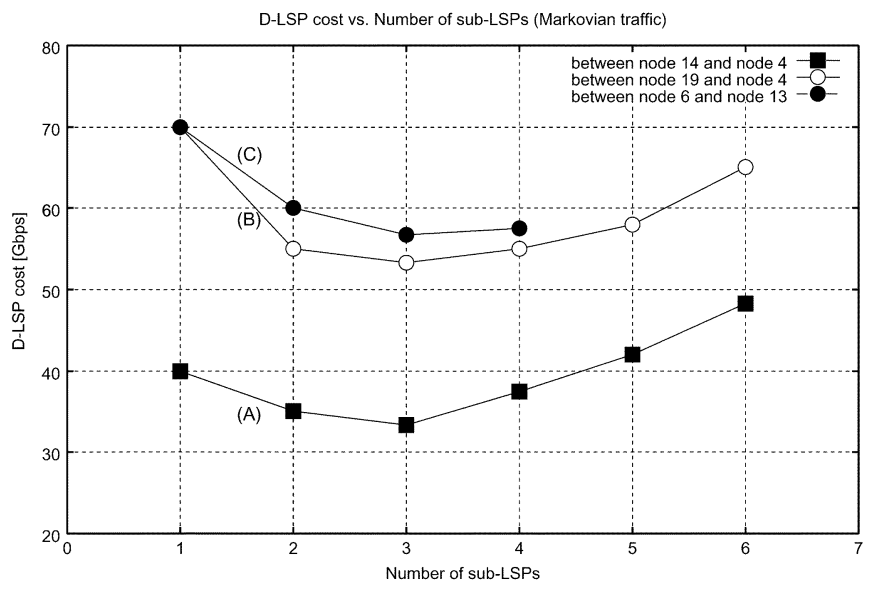

(a)

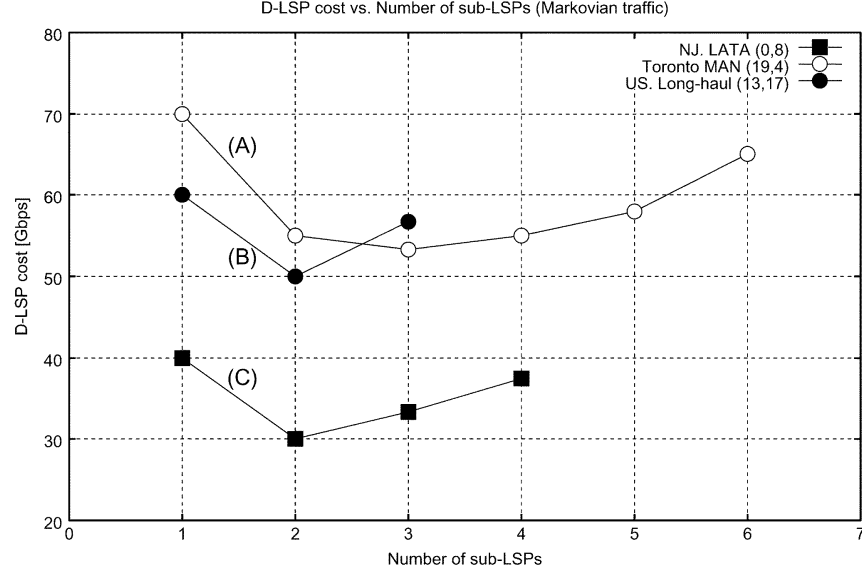

(b)

Fig. 5. D-LSP cost versus the number of sub-LSPs for Markovian traffic. (a) D-LSP cost curves of various node pairs in Toronto-MAN. (b) D-LSP cost curves in various example network topologies.

A series of curves in Fig. 6 shows that the trend in the networks of self-similar traffic is similar to that in the networks of Markovian traffic.

In the US-Longhaul network, there are some node pairs having only two link-disjoint routes, and there are node pairs between which the length of the third link-disjoint route is much longer than the length of the second route. Thus, LSP partitioning can result in a higher LSP cost than the cost of a single LSP. For these node pairs, the D-LSP cost-minimization algorithm establishes the protected D-LSP having a single sub-LSP and a backup LSP, as the conventional LSP scheme does.

Observing the trends of curves in Figs. 5 and 6 makes it obvious that the proposed D-LSP scheme is suitable for network serving either Markovian or self-similar traffic with consideration of statistical multiplexing. The D-LSP scheme can minimize the D-LSP cost with a moderate number of sub-LSPs. The D-LSP cost minimization algorithm can run fast with a computational complexity of $O(|R|)$. Usually $|R|$ is on the order of average node degree whose value is a moderate number, regardless of the network size. In order to evaluate the NCRR for various states in a given network, we consider a network-state parameter. The network activity defined as the ratio of the number of the "active" LER node pairs with LSP connections to the number of all possible node pairs in the network.

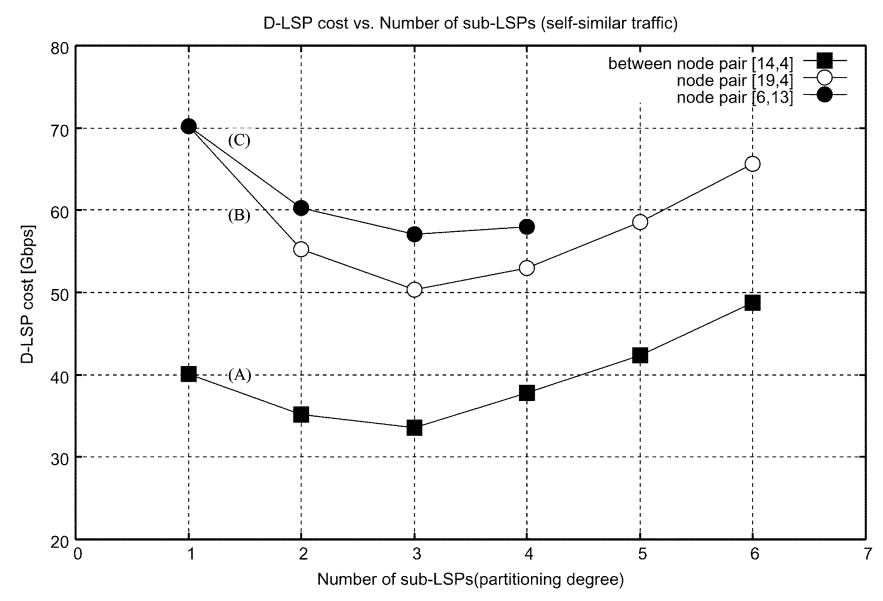

(a)

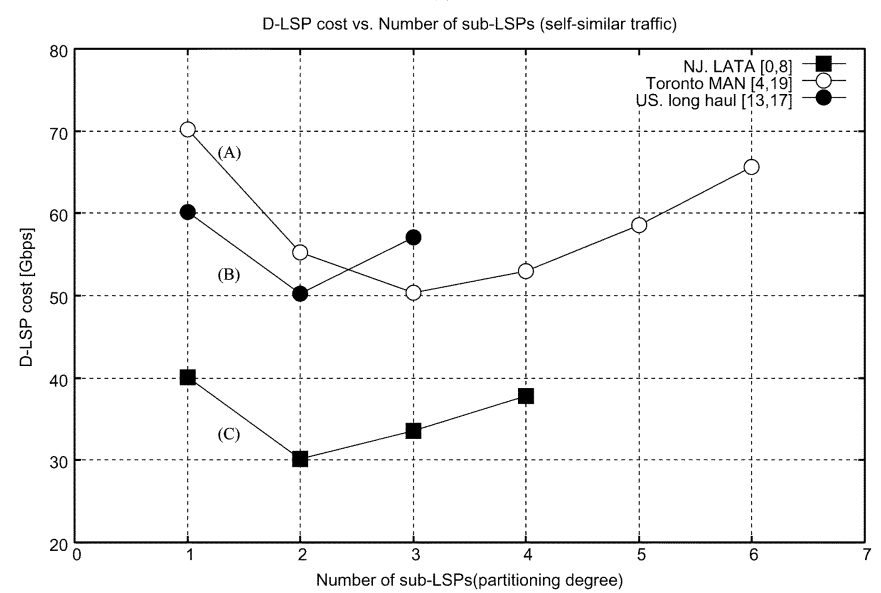

(b)

Fig. 6. D-LSP cost versus the number of sub-LSPs for self-similar traffic. (a) D-LSP cost curves of various node pairs in Toronto-MAN. (b) D-LSP cost curves in various example network topologies.

Fig. 7(a) shows that NCRR is at least $31 \%$ for the NJ-LATA, at least $27 \%$ for the Toronto-MAN, and at least $15 \%$ for the US-Longhaul, when these networks serve Markovian traffic. Fig. 7(b) shows that NCRR is at least $29 \%$ for the NJ-LATA, at least $27 \%$ for the Toronto-MAN, and at least $16 \%$ for the US-Longhaul when these networks serve self-similar traffic. Assuming that all links in the example network topologies have a constant weights, NCRR is not significantly affected by the network activity and the type of traffic model that the network serves.

\section{CONCLUSION}

A D-LSP scheme for reducing the cost of MPLS network protection is proposed. Uniform bandwidth distribution over sub-LSPs is shown to be the optimum for D-LSP cost minimization. The optimum number of sub-LSPs for each D-LSP is derived. The backup LSP of the D-LSP consisting of $K$ subLSPs requires only $1 / K$ of the amount of spare bandwidth required by the conventional LSP for fault restoration in a single link-failure scenario. The D-LSP scheme enhances network efficiency through reduction of the amount of spare bandwidth required and use of the proposed D-LSP cost minimization algorithm with a computational complexity of $O(|R|)$. In practical networks, $|R|$ is a moderate number, regardless of the network 


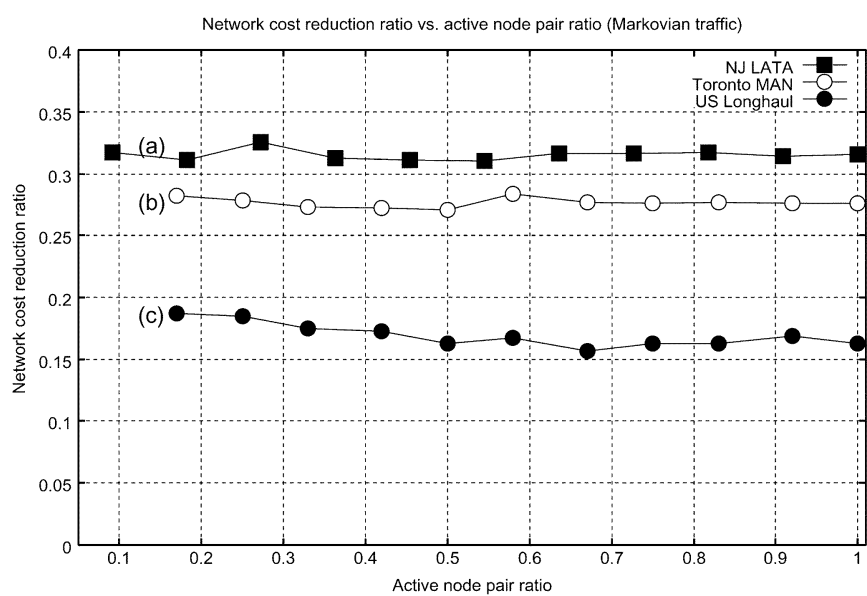

(a)

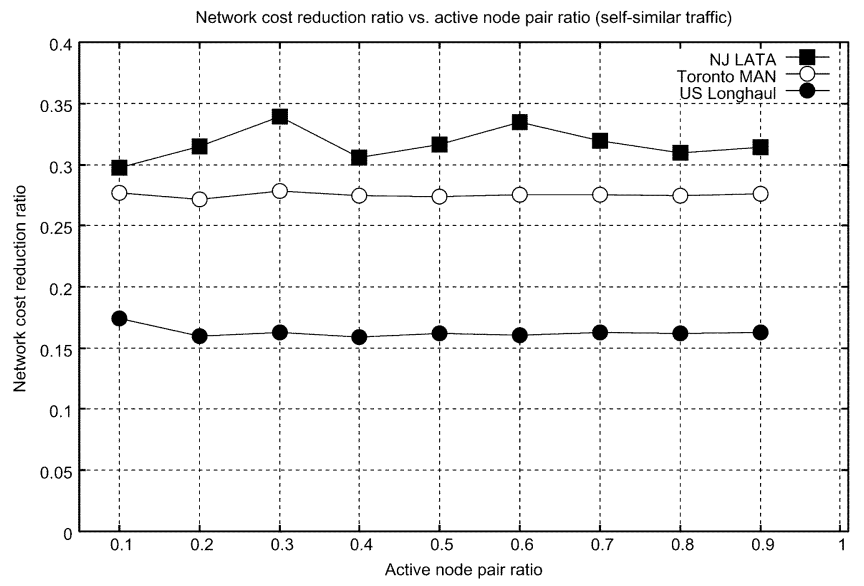

(b)

Fig. 7. NCRR between the conventional LSP and D-LSP schemes. (a) NCRR for Markovian traffic. (b) NCRR for self-similar traffic.

size. Hence, the D-LSP scheme can be a practical method for reducing the bandwidth cost of MPLS networks.

Numerical results show that the proposed D-LSP scheme significantly reduces the network cost in comparison with the conventional LSP scheme. NCRR is at least 29\%, 27\%, and $15 \%$ in example network topologies of which average node degree is $4.6,4.4$, and 3.2, respectively. The proposed D-LSP scheme is suitable for networks in which statistical multiplexing is considered. Numerical examples also show that the performance of the D-LSP scheme applied to Markovian traffic and self-similar traffic is quite similar in terms of NCRR. This means that the proposed D-LSP scheme is not affected significantly by the traffic patterns in backbone networks or MANs where a large number of packet flows are aggregated.

Network reliability will be further investigated for multiple failure scenarios in the D-LSP scheme.

\section{APPENDIX}

\section{A. Proof of Theorem 1}

Proof: For given $\mathbf{w}$, if we make $\mathbf{w}^{*}=\left(w_{1}^{*}, w_{2}^{*}, \ldots, w_{|R|}^{*}\right)$ by sorting the elements of $\mathbf{w}$ in descending order, then by Lemma 2

$$
C_{\mathrm{dlsp}}(\mathbf{w}) \geq C_{\mathrm{dlsp}}\left(\mathbf{w}^{*}\right)
$$

A new vector $\mathbf{w}^{\prime \prime}=\left(w_{1}^{\prime \prime}, w_{2}^{\prime \prime}, \ldots, w_{|R|}^{\prime \prime}\right)$ is defined such that $w_{i}^{\prime \prime}=w_{1}^{*}$ if $i \leq J, w_{J+1}^{\prime \prime}=D-J w_{1}^{*}=\alpha\left(0 \leq \alpha<w_{1}^{*}\right), w_{j}^{\prime \prime}=$ 0 for $j \geq J+1$, where $J=\left\lfloor(D) /\left(w_{1}^{*}\right)\right\rfloor$. Then, the following relation is derived as:

$$
\begin{aligned}
& C_{\mathrm{dlsp}}\left(\mathbf{w}^{\prime \prime}\right)-C_{\mathrm{dlsp}}\left(\mathbf{w}^{*}\right) \\
& \quad=\sum_{i=1}^{J}\left(w_{1}^{*}-w_{i}^{*}\right)\left|r_{i}\right|+\left(\alpha-w_{J+1}^{*}\right)\left|r_{J+1}\right|-\sum_{i=J+2}^{|R|} w_{i}^{*}\left|r_{i}\right| .
\end{aligned}
$$

If $\alpha>w_{J+1}^{*}$, the following relation holds:

$$
\begin{aligned}
& C_{\mathrm{dlsp}}\left(\mathbf{w}^{\prime \prime}\right)-C_{\mathrm{dlsp}}\left(\mathbf{w}^{*}\right) \\
& \leq \sum_{i=1}^{J}\left(w_{1}^{*}-w_{i}^{*}\right)\left|r_{J+1}\right| \\
&+\left(\alpha-w_{J+1}^{*}\right)\left|r_{J+1}\right|-\sum_{i=J+2}^{|R|} w_{i}^{*}\left|r_{J+1}\right| \\
&=\left|r_{J+1}\right|\left\{\sum_{i=1}^{J}\left(w_{1}^{*}-w_{i}^{*}\right)+\alpha-w_{J+1}^{*}-\sum_{i=J+2}^{|R|} w_{i}^{*}\right\} \\
&= 0 . \\
& \therefore C_{\mathrm{dlsp}}\left(\mathbf{w}^{\prime \prime}\right) \leq C_{\mathrm{dlsp}}\left(\mathbf{w}^{*}\right) .
\end{aligned}
$$

If $\alpha \leq w_{J+1}^{*}$, we can obtain the following:

$$
\begin{aligned}
C_{\mathrm{dlsp}}\left(\mathbf{w}^{\prime \prime}\right)-C_{\mathrm{dlsp}}\left(\mathbf{w}^{*}\right) \leq \sum_{i=1}^{J}\left(w_{1}^{*}-w_{i}^{*}\right)\left|r_{J}\right| \\
\quad+\left(\alpha-w_{J+1}^{*}\right)\left|r_{J}\right|-\sum_{J+2}^{|R|} w_{j}^{*}\left|r_{J}\right| \\
=\left|r_{J}\right|\left\{\sum_{i=1}^{J}\left(w_{1}^{*}-w_{i}^{*}\right)+\alpha-w_{J+1}^{*}-\sum_{j=J+2}^{|R|} w_{j}^{*}\right\} \\
=0 . \\
\therefore C_{\mathrm{dlsp}}\left(\mathbf{w}^{\prime \prime}\right) \leq C_{\mathrm{dlsp}}\left(\mathbf{w}^{*}\right) .
\end{aligned}
$$

From (A.1), (A.3), and (A.4), we can obtain the following inequality:

$$
C_{\text {dlsp }}\left(\mathbf{w}^{\prime \prime}\right) \leq C_{\text {dlsp }}(\mathbf{w}) .
$$

Since $\mathbf{w}^{\prime \prime} \in B$, the theorem is proved.

\section{B. Proof of Theorem 2}

Proof: Case 1: Suppose that the first relation is true. Then the proof is done regardless of the status (true or false) of the second relation.

Case 2: Suppose that the first relation is false. Then, subtraction of the left side from the right side of (11) is obtained as

$$
\frac{\alpha}{K}\left(\left|r_{1}\right|+\cdots+\left|r_{K}\right|-K\left|r_{K+1}\right|\right)>0 .
$$

From the definition of $\alpha$ in (10), we have

$$
\frac{D}{K+1}<w^{*}
$$


From (A.6) and (A.7), the subtraction of the left side from the right side of (12) is

$$
\left(\frac{D}{K+1}-w^{*}\right)\left(\left|r_{1}\right|+\cdots+\left|r_{K}\right|-K\left|r_{K+1}\right|\right)<0
$$

i.e., if (11) is false, then (12) is true.

From Cases 1 and 2, the proof is done.

\section{Proof of Theorem 3}

Proof: If there exists $\hat{k}$ such that $C_{\mathrm{dlsp}}(\hat{k}) \leq C_{\mathrm{dlsp}}(\hat{k}+1)$, then from (6)

$$
\left|r_{\hat{k}+1}\right| \geq \frac{1}{\hat{k}}\left\{\sum_{i=1}^{\hat{k}}\left|r_{i}\right|+\left|r_{\text {backup }}\right|\right\} .
$$

For $j \geq \hat{k}+1$, the following relation is derived as:

$$
\begin{aligned}
C_{\mathrm{dlsp}}(j)= & \frac{D}{j}\left\{\sum_{i=1}^{j}\left|r_{i}\right|+\left|r_{\text {backup }}\right|\right\} \\
\geq & \frac{D}{j}\left\{\sum_{i=1}^{\hat{k}}\left|r_{i}\right|+\left|r_{\text {backup }}\right|+(j-\hat{k})\left|r_{\hat{k}+1}\right|\right\} \\
\geq & \frac{D}{j}\left\{\sum_{i=1}^{\hat{k}}\left|r_{i}\right|+\left|r_{\text {backup }}\right|\right. \\
& \left.+(j-\hat{k}) \frac{1}{\hat{k}}\left\{\sum_{i=1}^{\hat{k}}\left|r_{i}\right|+\left|r_{\text {backup }}\right|\right\}\right\} \\
= & \frac{D}{\hat{k}}\left\{\sum_{i=1}^{\hat{k}}\left|r_{i}\right|+\left|r_{\text {backup }}\right|\right\}=C_{\mathrm{dlsp}}(\hat{k})
\end{aligned}
$$$$
\therefore C_{\mathrm{dlsp}}(j) \geq C_{\mathrm{dlsp}}(\hat{k}), \quad \forall j \geq \hat{k}
$$

\section{Proof of Theorem 4}

Proof: For given $\mathbf{n}$, if we make $\hat{\mathbf{n}}=\left(\hat{n}_{1}, \hat{n}_{2}, \ldots, \hat{n}_{|R|}\right)$ by sorting the elements of $\mathbf{n}$ in a descending order, then Lemma 3 gives the following relation:

$$
C_{\mathrm{dlsp}}(\hat{\mathbf{n}}) \leq C_{\mathrm{dlsp}}(\mathbf{n}) .
$$

Put $K=\left\lfloor N / \hat{n}_{1}\right\rfloor$. We introduce a new vector $\mathbf{n}^{\prime}=$ $\left(n_{1}^{\prime}, n_{2}^{\prime}, \ldots, n_{|R|}^{\prime}\right)$, where $n_{i}^{\prime}=\hat{n}_{1}$ for $j \leq K, n_{K+1}^{\prime}=$ $N-K \hat{n}_{1}$, and $n_{j}^{\prime}=0$ for $j \geq K+1$. Then, $\mathbf{n}^{\prime} \in \tilde{B}$. If we let $n_{K+1}^{\prime}=N-K \hat{n}_{1}=\xi$, we can obtain the following relation:

$$
\begin{aligned}
& C_{\mathrm{dlsp}}(\hat{\mathbf{n}})-C_{\mathrm{dlsp}}\left(\mathbf{n}^{\prime}\right) \\
& =\sum_{i=1}^{K}\left\{w^{\mathrm{eff}}\left(\hat{n}_{i}\right)-w^{\mathrm{eff}}\left(n_{i}^{\prime}\right)\right\}\left|r_{i}\right| \\
& \quad+\left\{w^{\mathrm{eff}}\left(\hat{n}_{K+1}\right)-w^{\mathrm{eff}}(\xi)\right\}\left|r_{K+1}\right| \\
& \quad+\sum_{i=K+2}^{|R|}\left\{w^{\mathrm{eff}}\left(\hat{n}_{i}\right)\right\}\left|r_{i}\right|
\end{aligned}
$$

$$
\begin{aligned}
& \geq \sum_{i=1}^{K}\left\{w^{\mathrm{eff}}\left(\hat{n}_{i}\right)-w^{\mathrm{eff}}\left(n_{i}^{\prime}\right)\right\}\left|r_{K+1}\right| \\
&+\left\{w^{\mathrm{eff}}\left(\hat{n}_{K+1}\right)-w^{\mathrm{eff}}(\xi)\right\}\left|r_{K+1}\right| \\
&+\sum_{i=K+2}^{|R|}\left\{w^{\mathrm{eff}}\left(\hat{n}_{i}\right)\right\}\left|r_{K+1}\right| \\
&=\left|r_{K+1}\right|\left[\sum_{i=1}^{|R|} w^{\mathrm{eff}}\left(\hat{n}_{i}\right)-\sum_{i=1}^{|R|} w^{\mathrm{eff}}\left(n_{i}^{\prime}\right)\right] \\
& \geq 0 .
\end{aligned}
$$

It can be easily shown that the last inequality of (A.12) is valid by applying Lemma 4 iteratively. By (A.11) and (A.12), the proof is done.

\section{E. Proof of Theorem 5}

Proof: Originally the domain of $C_{\mathrm{dlsp}}^{\prime}(n)$ is all integers in $[N /|R|, N]$. In order to make the analysis simple, we assume that the domain is the set of real numbers in $[N /|R|, N]$. If we let $U_{k}=(N /(k+1), N / k]$, then

$$
\left[\frac{N}{|R|}, N\right]=\left\{\frac{N}{|R|}\right\} \cup \bigcup_{k=1}^{|R|-1} U_{k} .
$$

Let us consider an interval $U_{k}$. For a real number $n \in$ $U_{k},\lfloor N / n\rfloor=k$, and $C_{\mathrm{dlsp}}^{\prime}(n)$ can be expressed as

$C_{\mathrm{dlsp}}^{\prime}(n)=\left\{\sum_{i=1}^{k}\left|r_{i}\right|+\left|r_{z}\right|\right\} w^{\mathrm{eff}}(n)+\left|r_{k+1}\right| w^{\mathrm{eff}}(N-k n)$.

The second derivative of $C_{\mathrm{dlsp}}^{\prime}(n)$ about $n$ is obtained as

$$
\begin{aligned}
\frac{d^{2} C_{\mathrm{dlsp}}^{\prime}(n)}{d n^{2}} & \\
= & -\left\{\sum_{i=1}^{k}\left|r_{i}\right|+\left|r_{z}\right|\right\} \frac{2 \alpha^{2} b^{2}(1-\rho)^{3} r d}{(d n+\alpha b(1-\rho))^{3}} \\
& -\left|r_{k+1}\right| \frac{2 k^{2} \alpha^{2} b^{2}(1-\rho)^{3} r d}{(d(N-k n)+\alpha b(1-\rho))^{3}} .
\end{aligned}
$$

For $n \in U_{k}, N-k n \geq 0$ and thus, the value of (A.13) is negative. Since $C_{\mathrm{dlsp}}^{\prime}(n)$ is concave in $U_{k}$, the minimum value of $C_{\mathrm{dlsp}}^{\prime}(n)$ exists at either end point of $U_{k}$. However, returning to the original condition, we are interested only in the integer values of $n$ in $U_{k}$. In case of considering integers in $U_{k}$, the minimum value of $C_{\mathrm{dlsp}}^{\prime}(n)$ exists at either $n=\lfloor N /(k+1)\rfloor+1$ or $n=\lfloor N / k\rfloor$. Thus, global minimum of $C_{\mathrm{dlsp}}^{\prime}(n)$ exists among $\left\{C_{\mathrm{dlsp}}^{\prime}(n): n \in \mathcal{N}_{0} \cup \mathcal{N}_{1}\right\}$.

\section{F. Proof of Lemma 6}

Proof: In order to use Lemma 5, we assume that the parameter $n$ in $w^{e}(n)$ is continuous. It can be shown by the implicit function theorem [19] that $w^{e}(n)$ is continuously differentiable, and an implicit formula of $w^{e \prime}(n)$ is obtained for positive $n$. Using this formula, we can also show that $w^{e^{\prime}}(n)$ is continuous, and $w^{e}(n)$ is twice differentiable for positive $n$. Thus, by Lemma 5, it is sufficient to show that $w^{e \prime \prime}(n)<0$. By changing 
the variable $n$ to $x$ in (18) and differentiating it twice with respect to $x$, we have

$$
\begin{aligned}
w^{e \prime \prime}(x)=\frac{A \sigma^{1 / H} x^{1 / 2 H-2} w^{e}(x)}{\left(w^{e}(x)\right)^{p+1}+A \sigma^{1 / H} p} \frac{p+1}{2} & \\
& \times \frac{1}{\left\{(1+p) w^{e}(x)-x m p\right\}^{2}} g(x)
\end{aligned}
$$

where $A=\left(E\left[Q^{*}\right] / d\right)^{p}$ and

$$
g(x)=-\frac{1}{2}(p+1)^{2}\left\{w^{e}(x)+\frac{p m x}{p+1}\right\}^{2}+\frac{1}{2} p m^{2} x^{2} .
$$

In the above equation, we can know that all other terms comprising $w^{e \prime \prime}(x)$ are positive except $g(x)$, since $w^{e}(x) \geq 0$. Since $w^{e}(x) \geq 0$, we can know that $w^{e}(x) \geq x m$ from (18). This makes sense, because the service rate should not be less than the average traffic arrival rate, in order to guarantee any QoS. Under this range of $w^{e}(x)$, we can show that $g(x)$ is bounded by

$$
g(x) \leq-\frac{1}{2}\left(4 p^{2}+3 p+1\right) m^{2} x^{2} .
$$

Thus, for $x>0, g(x)<0$, and $w^{e \prime \prime}(x)<0$.

\section{G. Proof of Theorem 6}

Proof: In order to make the analysis simple, we assume that the domain of $C_{\mathrm{dlsp}}^{\prime}(n)$ is the set of real numbers in $[N /|R|, N]$. If we let $U_{k}=(N /(k+1), N / k]$, then

$$
\left[\frac{N}{|R|}, N\right]=\left\{\frac{N}{|R|}\right\} \cup \bigcup_{k=1}^{|R|-1} U_{k} .
$$

Let us consider an interval $U_{k}$. For a real number $n \in$ $U_{k},\lfloor N / n\rfloor=k$ and $C_{\text {dlsp }}^{\prime}(n)$ can be expressed as

$$
C_{\mathrm{dlsp}}^{\prime}(n)=\left\{\sum_{i=1}^{k}\left|r_{i}\right|+\left|r_{z}\right|\right\} w^{e}(n)+\left|r_{k+1}\right| w^{e}(N-k n) .
$$

The second derivative of $C_{\mathrm{dlsp}}^{\prime}(n)$ about $n$ is obtained as

$$
\begin{aligned}
\frac{d^{2} C_{\mathrm{dlsp}}^{\prime}(n)}{d n^{2}}=\left\{\sum_{i=1}^{k}\left|r_{i}\right|\right. & \left.+\left|r_{z}\right|\right\} \\
& \times w^{e \prime \prime}(n)+\left|r_{k+1}\right| k^{2} w^{e \prime \prime}(N-k n) .
\end{aligned}
$$

Since we already showed that $w^{e \prime \prime}(n)<0$ for $n>0$ in Lemma 6 , the second derivative of $C_{\mathrm{dlsp}}^{\prime}(n)$ is also negative. Hereafter, by the same reasoning as Theorem 5 , the proof is completed.

\section{REFERENCES}

[1] Requirement for traffic engineering over MPLS, IETF RFC 2702, Sep. 1999, D. Awduche.

[2] Multiprotocol label switching architecture, IETF RFC 3031, Jan. 2001, E. Rosen.

[3] Framework for MPLS-based recovery, IETF Draft-Ietf-MPLS-Recovery-Framework-06.txt, July 2002, V. Sharma.

[4] C. Huang, V. Sharma, K. Owen, and S. Makam, "Building reliable MPLS networks using a path protection mechanism," IEEE Commun. Mag., vol. 40, no. 3, pp. 156-162, Mar. 2002.

[5] R. R. Iraschko, M. H. MacGregor, and W. D. Grover, "Optimal capacity placement for path restoration in STM or ATM mesh-survivable networks," IEEE/ACM Trans. Netw., vol. 6, no. 3, pp. 325-336, Jun. 1998.

[6] Y. Liu, D. Tipper, and P. Siripongwutikorn, "Approximating optimal spare capacity allocation by successive survivable routing," in Proc. IEEE INFOCOM, 2001, pp. 699-708.

[7] W. D. Grover, V. Rawat, and M. H. MacGregor, "Fast heuristic principle for spare capacity placement in mesh-restorable SONET/SDH transport networks," Electron. Lett., vol. 33, no. 3, pp. 195-196, Jan. 1997.

[8] Y. Katsube and T. Kodama, "QoS control in an ATM network considering virtual path concept," in Proc. IEEE GLOBECOM, 1990, pp. 1104-1110.

[9] S. H. Kang and D. K. Sung, "A CAC scheme based on real-time cell loss extimation for ATM multiplexers," IEEE Trans. Commun., vol. 48 , no. 2, pp. 252-258, Feb. 2000.

[10] R. Guerin, H. Ahmadi, and M. Naghshineh, "Equivalent capacity and its application to bandwidth allocation in high-speed networks," IEEE J. Sel. Areas Commun., vol. 9, no. 7, pp. 968-981, Sep. 1991.

[11] R.-H. Hwang, J. F. Kurose, and D. Towsley, "MDP routing in ATM networks using virtual path concept," in Proc. IEEE INFOCOM, Jun. 1994, vol. 3, pp. 1509-1517.

[12] M. E. Crovella and A. Bestavros, "Self-similarity in world wide web traffic: Evidence and possible causes," IEEE/ACM Trans. Netw., vol. 5, no. 6, pp. 835-846, Dec. 1997.

[13] W. E. Lenald, M. S. Taqqu, W. Willinger, and D. V. Wilson, "On the self-similar nature of Ethernet traffic," IEEE/ACM Trans. Netw., vol. 2, no. 1, pp. 1-15, Feb. 1994.

[14] V. Paxson and S. Floyd, "Wide-area traffic: The failure of Poisson modeling," IEEE/ACM Trans. Netw., vol. 3, no. 3, pp. 226-244, Jun. 1995.

[15] S. Kim, S. Y. Nam, and D. K. Sung, "Effective bandwidth for a single server queueing system with fractional Brownian input," Perform. Eval., vol. 61, pp. 203-223, Jul. 2005.

[16] H. Sakauchi, Y. Nishimura, and S. Hasegawa, "A self-healing network with an economical spare channel assignment," in Proc. IEEE GLOBECOM, 1990, pp. 438-443.

[17] W. D. Grover, "The self healing network: A fast distributed restoration technique for networks using digital cross connect machines," in Proc. IEEE GLOBECOM, 1987, pp. 1090-1095.

[18] R. Bhandari, Survivable Networks: Algorithms for Diverse Routing. Norwell, MA: Kluwer, 1999.

[19] W. Kaplan, Advanced Calculus, 4th ed. Reading, MA: Addison-Wesley, 1991.

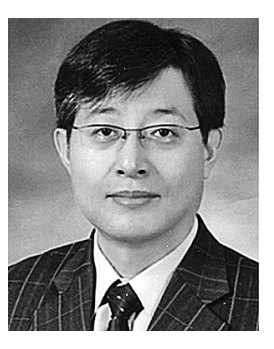

Kyu-Seek Sohn (S'02-M'03) received the B.S and M.S. degrees in electronics engineering from Hanyang University, Seoul, Korea, in 1982, and 1984, respectively; and the Ph.D. degree in electrical engineering from the Korea Advanced Institute of Science and Technology (KAIST), Daejeon, Korea, in 2003.

From December 1984 to March 2002, he was a Research Engineer with LG Cable Ltd., Seoul, Korea, where he had been engaged in various projects including the development of a fiber optic outside plant operation and management system. In 2004, he joined the faculty of Hanyang Cyber University, Seoul, Korea, where he is currently a full-time Lecturer with the Department of Information and Communications. His research interests include performance and reliability of communication systems and networks, high-speed networks, and optical access networks. 


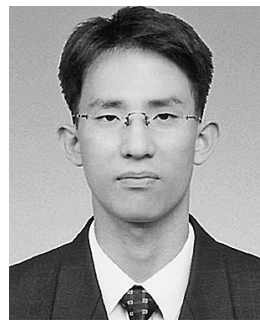

Seung Yeob Nam (S'97-A'04-M'05) received the B.S., M.S., and Ph.D. degrees in electrical engineering from the Korea Advanced Institute of Science and Technology (KAIST), Daejon, Korea, in 1997, 1999, and 2004, respectively.

He is currently a Postdoctoral Research Fellow with CyLab, Carnegie Mellon University, Pittsburgh, PA, supported by both CyLab and the Postdoctoral Fellowship Program of the Korea Science and Engineering Foundation (KOSEF), where he is working in the area of internet security and survivable networks. His research interests include network performance monitoring, scalable provisioning of QoS in the internet, internet security, survivable networks, high-speed switching systems, wireless networks, etc.

Dr. Nam received the Best Paper Award from the APCC 2000 Conference and the Bronze Prize from the 2004 Samsung Humantech paper contest. He is a Member of ACM.

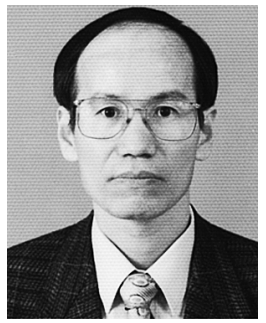

Dan Keun Sung (S'80-M'82-SM'00) received the B.S. degree in electronics engineering from Seoul National University, Seoul, Korea, in 1975, and the M.S. and Ph.D. degrees in electrical and computer engineering from the University of Texas at Austin, in 1982 and 1986, respectively.

In 1986, he joined the faculty of the Korea Advanced Institute of Science and Technology (KAIST), Daejeon, Korea, where he is currently a Professor with the Department of Electrical Engineering and Computer Science. He was Director of the Satellite Technology Research Center (SaTReC) of KAIST from 1996 to 1999. His research interests include mobile communication systems and networks, high-speed networks, next-generation IP-based networks, traffic control in wireless and wireline networks, signaling networks, intelligent networks, performance and reliability of communication systems, and microsatellites.

Dr. Sung is an Editor of IEEE Communications Magazine. He is also Division Editor of the Journal of Communications and Networks. He received the National Order of Merits, Dongbaek Medal in 1992, Research Achievement Award in 1997, MoMuc Paper Award in 1997, Academic Excellence Award in 2000, Best Paper Award from APCC2000, and This Month's Scientist Award by MOST and KOSEF in 2004. He is a Member of the National Academy of Engineering of Korea. 